\title{
A feasibility study on ECT monitoring of phase migration in porous solids
}

\author{
S. Liu ${ }^{1}$, J. Liu ${ }^{2} \&$ Q. Chen ${ }^{3}$ \\ ${ }^{1}$ School of Energy and Power Engineering, \\ North China Electric Power University, Beijing 102206, China \\ ${ }^{2}$ Institute of Engineering Thermophysics, CAS, Beijing 100080, China \\ ${ }^{3}$ School of Mechanics and Control Engineering, \\ Beijing Jiaotong University, Beijing 100044, China
}

\begin{abstract}
In the study of the strength of soil/sand, and the cause of the incipience of debris flow, it is important to monitor the distribution and the migration of ice and water contents in such porous media. To overcome the difficulty of "seeing" through opaque soil, an experimental investigation was conducted to explore the feasibility of non-intrusive monitoring of ice migration in porous media by using electric capacitance tomography (ECT). A ten-electrode sensor was made for signal acquisition and OIOR method was used for image reconstruction. The distribution and penetration of ice in porous solids are displayed clearly by ECT images. ECT data were also validated against the temperature data measured by thermocouples, and the feasibility of such ECT method is convincingly proven.
\end{abstract}

Keywords: flow monitoring, flow in porous solids.

\section{Introduction}

Ice penetration or melting in soil/sand (porous solids) can significantly alter the stability of the solid matrix, which will affect the possibility of debris/rock flow. Therefore, it is important to understand the relationship between ice penetration or melting in soil and the corresponding change in the mechanical properties of the soil. However, it is difficult to monitor ice and water contents and their migration in such porous media by conventional techniques, due to the limited sensing accessibility, e.g. opacity, of the solids.

Electrical Capacitance Tomography (ECT) is a recently developed nonintrusive technique for visualizing the distribution of materials. As an example, 


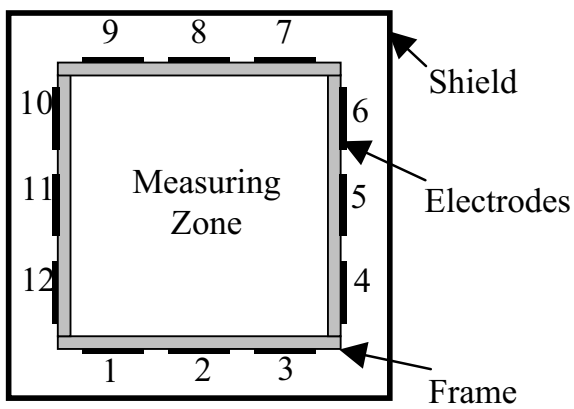

Figure 1: $\quad$ ECT sensor.

figure 1 is a sketch of a twelve-electrode ECT sensor. The electrodes are numbered from 1 to 12 . Inside the frame is the measuring zone. Surrounding the electrodes is a metal shield. ECT does not disturb the process inside the measuring zone, and images are produced via image reconstruction algorithms [1]. It is the purpose of this study to examine the feasibility of ECT to visualize the distribution and migration of ice and water contents in porous solids, aiming at further development of ECT into a viable monitoring technique for the study of ice penetration or melting in soil.

For a sensor of $\mathrm{x}$ electrodes, the number of independent capacitance measurements is $m=C_{x}^{2}=x(x-1) / 2$ and the measured capacitances are represented by an array $\mathbf{C}=\left(\mathrm{C}_{1}, \mathrm{C}_{2}, \ldots, \mathrm{C}_{\mathrm{m}}\right)^{\mathrm{T}}$. An ECT image is reconstructed based on the values of the capacitances. Usually a measuring zone is virtually divided into, say $n$, pixels, then the distribution of the objects in the measuring zone is represented by an array of grey values on the pixels, i.e. $\mathbf{G}=\left(\mathrm{G}_{1}, \mathrm{G}_{2}, \ldots\right.$, $\left.G_{n}\right)^{T}$, where $G_{i}$ is the grey value on pixel $i$.

One of the most earlier and common image reconstruction method is Linear Back Projection, LBP, in which a linear relationship between $\mathrm{C}$ and $\mathrm{G}$ is assumed:

$$
\mathbf{C}=\mathbf{S G}
$$

where $\mathbf{S} \in R^{m \times n}$ is a coefficient matrix, commonly called sensitivity map. The descriptions of the methods to derive $\mathbf{S}$ are abundant in the literature, e.g. Xie et al. [2].

LBP reconstructs images according to:

$$
\mathbf{G}=\mathbf{S}^{T} \mathbf{C}
$$

where $\mathbf{S}^{\mathrm{T}}$ is the transpose of $\mathbf{S}$.

LBP is simple and fast, but often suffers from the problems of notorious "illposed" nature, i.e. the severe deficit of the measured data with regard to the large number of pixels. The quality of LBP is often poor.

To improve the quality of the images, various image reconstruction techniques have been developed over the years [3-6]. Among them iterative methods have been widely applied. 
A most widely applied iterative method is the Landweber [3] method that repeatedly applies equation (1) (forward process) and equation (2) (inverse process) to gradually reduce the errors, as described by:

$$
\begin{gathered}
\mathbf{G}_{0}=\mathbf{S}^{\mathrm{T}} \mathbf{C} \\
\mathbf{G}_{k+1}=\mathbf{G}_{k}+\alpha_{k} \mathbf{S}^{\mathrm{T}}\left(\mathbf{C}-\mathbf{S} \mathbf{G}_{k}\right)=\mathbf{G}_{k}+\alpha_{k} \mathbf{S}^{\mathrm{T}} \mathbf{e}_{k}, \quad(k=0,1,2, \cdots) \\
\mathbf{G}_{\text {final }}=\mathbf{G}_{\text {final }-1}, \text { when } \mathbf{e}^{(\mathrm{k})}<\text { certain criterion }
\end{gathered}
$$

Due to the correction in each step, the new images are expected to gradually approach the true image. The process continues until a certain criterion is met, e.g. $\left\|\left(\mathbf{C}-\mathbf{S G}_{k}\right)\right\| /\|\mathbf{C}\|<0.01$.

However, an inherit drawback of the iterative methods is the cost of time. It has long been desired for an ECT system to perform "online iteration", i.e. to take measurements with the quality of an iterative method whilst maintaining the advantage of fast speed. For this, a new method, namely OIOR (OfflineIteration-Online-Reconstruction), has been developed by the authors [7].

OIOR has two parts, in the first part the iterations are performed offline to obtain a matrix $\mathbf{D}_{z}$ of the same dimensions as the transpose of the sensitivity map: i.e.

$$
\begin{gathered}
\mathbf{D}_{0}=\mathbf{S}^{T} \\
\mathbf{D}_{k+1}=\mathbf{A}_{k} \mathbf{D}_{k}+\mathbf{B}_{k}=\left(\mathbf{I}-\alpha_{k} \mathbf{S}^{T} \mathbf{S}\right) \mathbf{D}_{k}+\alpha_{k} \mathbf{S}^{T} \\
=\mathbf{D}_{k}+\alpha_{k} \mathbf{S}^{T}\left(\mathbf{I}-\mathbf{S D}_{k}\right) \quad(k=1,2, \cdots, z)
\end{gathered}
$$

in which $\mathbf{I} \in \mathbf{R}^{m \times m}$ is an identity matrix.

In the second stage an images is reconstructed in the same way as LBP, but using matrix $\mathbf{D}_{z}$ :

$$
\mathbf{G}=\mathbf{D}_{\mathrm{z}} \mathbf{C}
$$

Equation (8) is of exactly the same form as LBP, meaning that the algorithm will have the same image reconstruction speed as LBP, i.e. it can be used for online measurement like LBP does. However, as $\mathbf{D}_{z}$ is the result after many iterations, equation (8) will yield improved image quality as an iterative method does. Therefore, the new algorithm is effectively an online iterative image reconstruction method.

\section{Experimental}

\subsection{Test apparatus}

To study ice propagation in porous solids, a test unit is built shown in figure 2 . The centre zone, depicted by A-B-D-C, is the test zone wherein the ice propagation occurs. Temperatures on the upper cover and the lower cover are controlled individually by circulating a liquid coolant, so that a temperature gradient can be maintained at desired values. 
As the top and the bottom surfaces are unavailable for ECT electrodes, the ECT sensor has to be in an unclosed structure with electrodes arranged only on the two sides, depicted in figure 2. The ECT sensor has ten electrodes, five on each side, with a radial guard placed between each pair of electrodes. An insulating wrap covers the sensor. This unique feature requires a specially designed sensitivity map for image reconstruction.

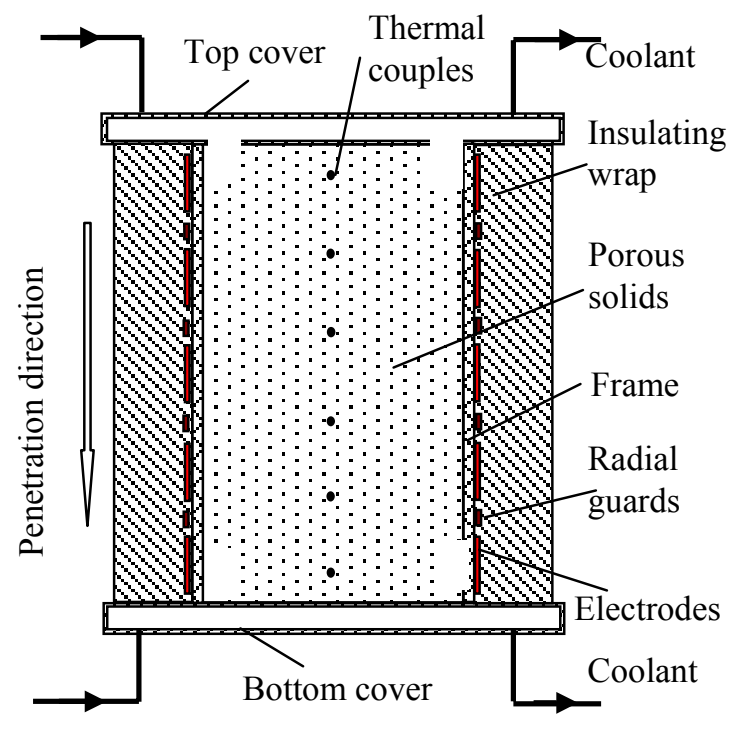

Figure 2: $\quad$ Sketch of the test unit.

There are also six thermocouples placed in the test medium, all along the central line, to provide some referencing indications of the temperature distributions inside.

The ECT device is an AC based system, comprised of the sensor, the data acquisition electronics, and a computer for data processing, as depicted in figure 3. The excitation voltage is a $15 \mathrm{~V}$ sine wave with a frequency of $200 \mathrm{KHz}$. Data collection speed is can be adjusted up to 140 frames per second. More details about the device can be found in [1].

\subsection{Experimental procedures}

The test medium, namely sand, was first dried thoroughly in an oven. Then a proper amount of water was fully mixed with the solids to achieve desired moisture content. In this study, 5\% water content was selected for all the experiments. The test medium was then sealed in a glass jar for 24 hours to reach uniform distribution of the moisture in the solids. After that, the test medium was filled into the test section and temperatures at the top and bottom covers were adjusted to the desired temperature gradient. Ice then began to form and ice front started to move from the top cover to the bottom cover. 


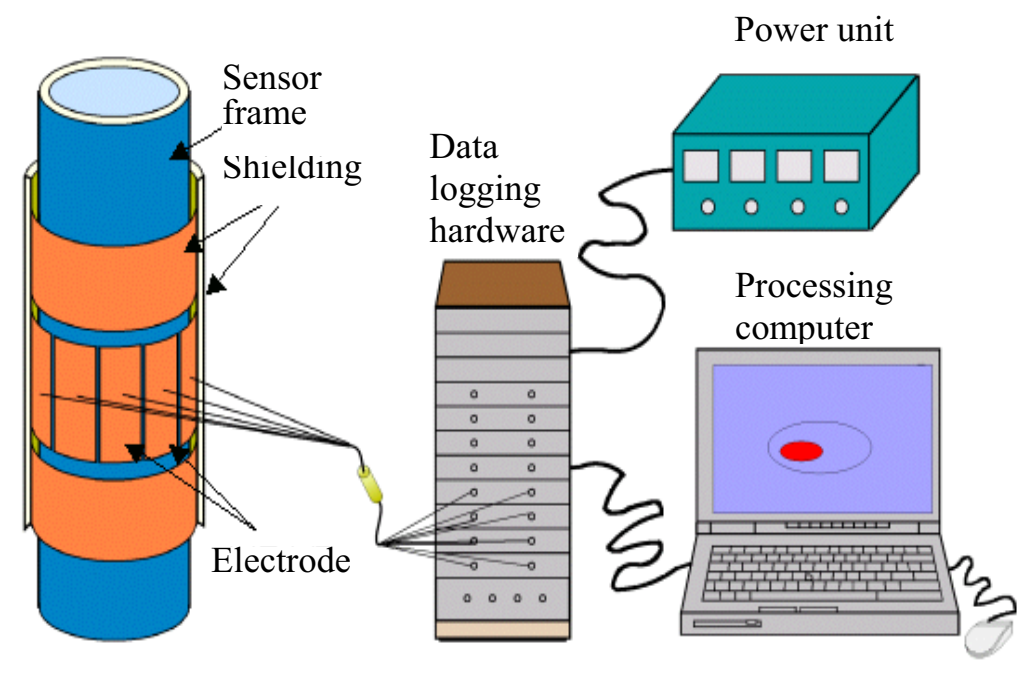

Figure 3: $\quad$ ECT system.

ECT measurements were then taken to image the ice distribution and motion inside the measuring zone. OIOR method, i.e. equation (8), was used for image reconstruction. As ice movement was slow, a 10 frame/second data acquisition speed would be sufficient.

\section{Results and discussions}

Figures 4-9 (a)s and (b)s show respectively the 2D and 3D images acquired by ECT, while (c)s show the temperatures profile along the centre line. Because the experiment begins with purely wet sand in the test zone, therefore, the permittivity is the highest in figure 4 (a) and (b), and the grey levels of the pixels of the image are generally high. When frozen zone forms, the permittivity decreases and the grey levels of the image pixels reduce in zone I, see figure 5 (a) and (b). As the frozen zone extends deeper, the low permittivity zone follows as well, reflected by the lighter color in (a)s and lower surfaces in (b)s of the figures. This continues until ice zone occupies almost all the test zone, shown in figure 9. In figures 5-8 (a)-(b) it can be seen that after a certain length of time, i.e. $3.3,3.8,5.5$ and 5.9 hour respectively, the tip of the ice front correspondingly reaches positions roughly $58 \mathrm{~mm}$ (red dotted line), 50, 35 and $20 \mathrm{~mm}$ above the bottom.

The temperature profiles measured by the thermocouples are used to compare with the ECT results. Figures 4-9 (c)s show temperature distribution alone the freezing direction, i.e. the axial line of the test zone. Before freezing takes place, the temperature is about $20.4^{\circ} \mathrm{C}$ at all the points, and the temperatures drop after freeing. In fact, the freezing point corresponding to $5 \%$ water content is $-0.42{ }^{\circ} \mathrm{C}$, as indicated by the dotted lines in the figures. Therefore, judging from the 
46 Monitoring, Simulation, Prevention and Remediation of Dense Debris Flows II

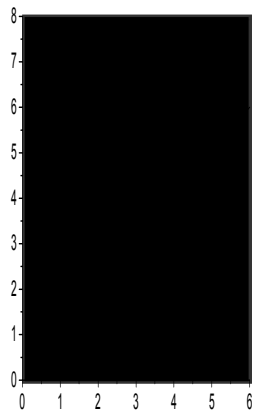

(a)

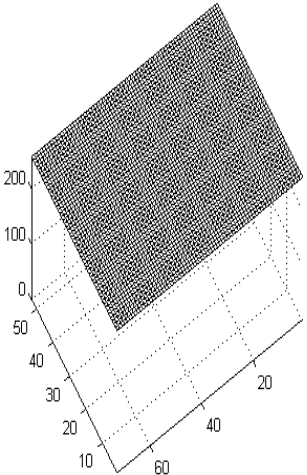

(b)

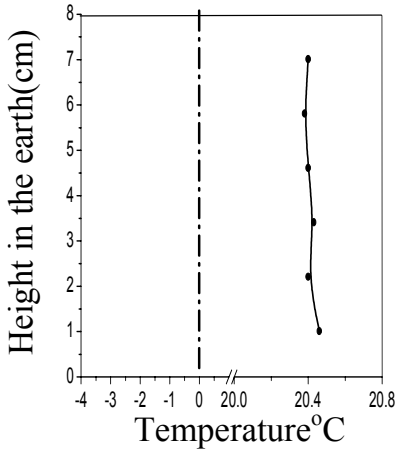

(c)

Figure 4: $\quad$ ECT images and temperature before freezing $(\mathrm{t}=0 \mathrm{~h})$

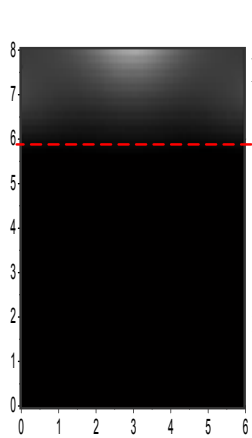

(a)



(b)

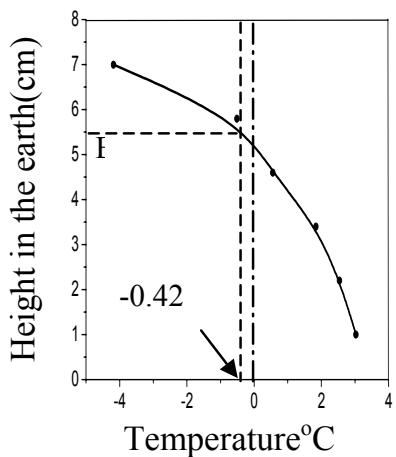

(c)

Figure 5: $\quad$ ECT images and temperature profile when $\mathrm{t}=3.3 \mathrm{~h}$.

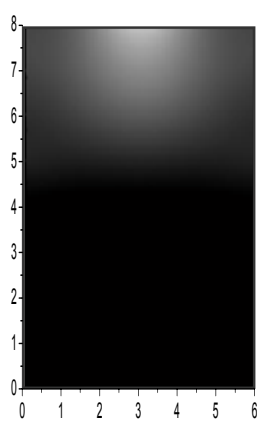

(a)

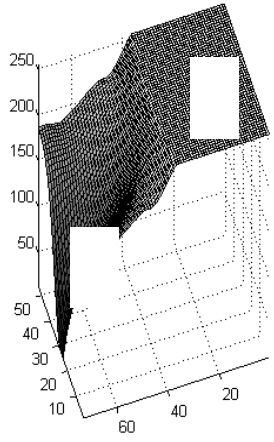

(b)

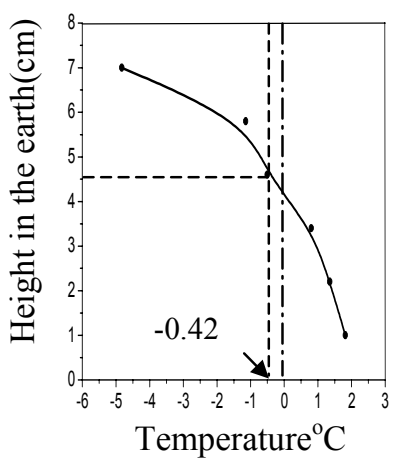

(c)

Figure 6: $\quad$ ECT images and temperature profile when $\mathrm{t}=3.8 \mathrm{~h}$. 
Monitoring, Simulation, Prevention and Remediation of Dense Debris Flows II 47



(a)

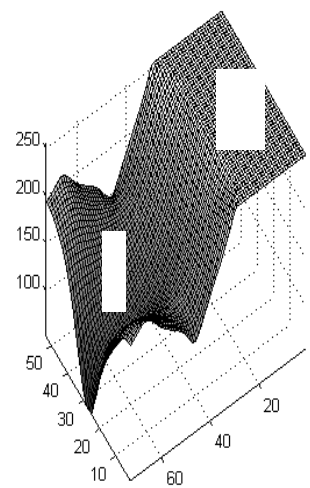

(b)



(c)

Figure 7: $\quad$ ECT images and temperature profile when $\mathrm{t}=5.5 \mathrm{~h}$.

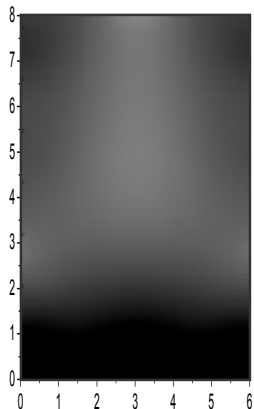

(a)

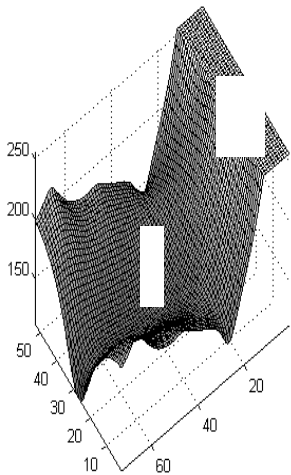

(b)

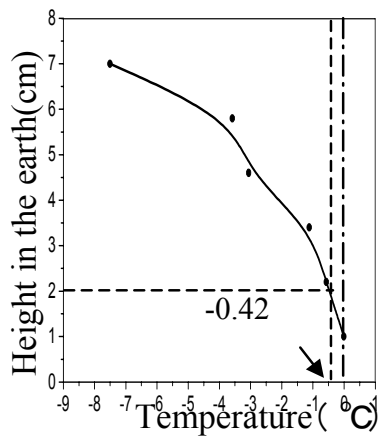

(c)

Figure 8: $\quad$ ECT images and temperature profile when $\mathrm{t}=5.9 \mathrm{~h}$.

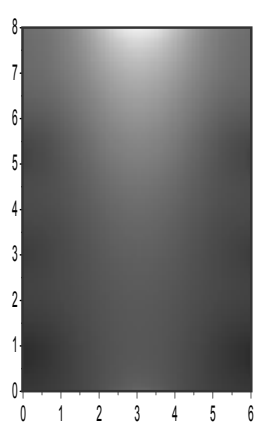

(a)

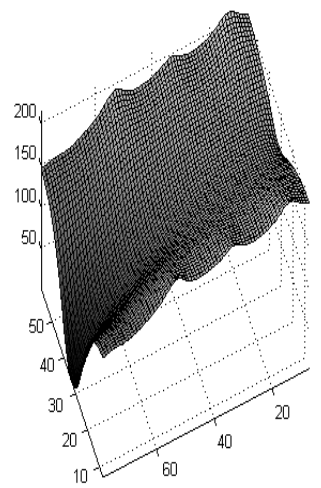

(b)

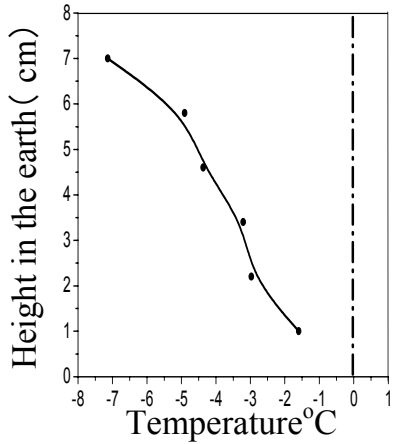

(c)

Figure 9: $\quad$ ECT images and temperature profile when $\mathrm{t}=7.8 \mathrm{~h}$. 
figures, it can been seen that the ice front researches approximately 54, 46, 29, and $20 \mathrm{~mm}$ above the bottom cover, when time span is $3.3,3.8,5.5$, and 5.9 hours respectively.

\section{Conclusions}

ECT measurements of ice distribution and motion in porous materials have been successful in the current investigation. The non-intrusive measuring method of ECT can be advantageous for materials of opaque nature, and enables monitoring of material distributions inside the measuring zone without disturbing the real condition.

ECT data are also compared with the temperature measurements by thermocouples and reasonably good agreements are obtained, which proves the feasibility of ECT method for the current problems.

As such a method is new and still in its developing stage, more studies are needed to gain detailed understanding both of ECT measurement and the mechanism of ice motion in soil.

\section{Acknowledgements}

The authors wish to express their gratitude to CAS (KZCX2-YW-302) and NSFC $(60672151,60532020)$ for sponsoring this study.

\section{References}

[1] Liu, S., Wang, H. G., Jiang, F. \& Yang, W. Q., A new image reconstruction method for tomographic investigation of fluidized beds, $A I C h E J, \mathbf{4 8}$, pp. 1631-1638, 2002.

[2] Xie, C., Huang, S., Hoyle, B. S., Thorn, R., Lenn, C., Snowden, D. \& Beck, M. S., Electrical capacitance tomography for flow imaging: system model for development of image reconstruction algorithms and design of primary sensors, in IEE Proc.-G, 139, pp. 89-97,. 1992.

[3] Landweber, L., An iterative formula for Fredholm integral equations of the first kind, Amer. J. Math,73, pp. 615-624, 1951.

[4] Yang, W. Q. \& Peng, L. H., Image reconstruction algorithms for electrical capacitance tomography, Meas. Sci. Technol., 14, pp. R1-R13, 2003.

[5] Liu, S., Fu, L. \& Yang, W. Q., Optimization of iterative image reconstruction process for electrical capacitance tomography, Meas. Sci. Technol., 10, pp. L37-L39, 1999.

[6] Isaksen, $\varnothing$. \& Nordtvedt, J. E., A new reconstruction algorithm for process tomography, Meas. Sci. and Technol., 4, pp. 1464-1475, 1993.

[7] Liu, S., Fu, L., Yang, W. Q., Wang, H. \& Jiang, F., On-line Iterative Image Reconstruction for ECT, Proceedings of the $3^{\text {rd }}$ World Congress on Industrial Process Tomography, Banff, Canada, pp. 403-408, 2003. 